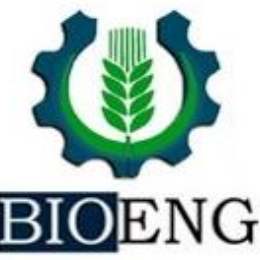

\title{
CLUSTERS DE ROBUSTEZ COMO CRITÉRIO DE SELEÇÃO NO MELHORAMENTO GENÉTICO PARA MITIGAÇÃO DE IMPACTOS DAS MUDANÇAS CLIMÁTICẢS
}

\author{
R. N. Pereira ${ }^{1 *}$, R. L. Serodio $^{1}$, H. T. Ventura $^{2}$, F. R. Araújo Neto ${ }^{3}$, \\ N. T. Pegolo ${ }^{1}$
}

${ }^{1}$ Instituto Federal de Educação, Ciência e Tecnologia de São Paulo, Campus Avaré, SP, Brasil

${ }^{2}$ Associação Brasileira de Criadores de Zebu, Uberaba, MG, Brasil

${ }^{3}$ Instituto Federal de Educação, Ciência e Tecnologia Goiano, Campus Rio Verde, GO, Brasil

Article history: Received 08 April 2018; Received in revised form 10 June 2018; Accepted 13 June 2018 ; Available online 28 June 2018.

\section{RESUMO}

Mudanças climáticas são previstas para as próximas décadas e, consequentemente, seus impactos na pecuária bovina, sendo a seleção nos rebanhos uma maneira de amenizá-los. Este trabalho teve como objetivo desenvolver um sistema de seleção baseado nos parâmetros genéticos gerados por modelos de norma de reação adaptativa em bovinos da raça Nelore. Foram utilizados dados genealógicos e de crescimento fornecidos pela Associação Brasileira de Criadores de Bovinos. Definiu-se um gradiente ambiental baseado em valores médios de grupos contemporâneos padronizados. Para a predição de coeficientes das normas de reação adaptativas utilizou-se a regressão aleatória com polinômios cúbicos para pesos aos 450 dias com análise de sexos separados. Foram calculados os valores genéticos dos diferentes indivíduos em função de um gradiente ambiental utilizando o software BLUPF90. Os indivíduos foram classificados considerando coeficientes que gerassem normas com valores genéticos elevados e com menor variação ao longo do gradiente ambiental. Compensou-se, então, a elevação do valor genético e a sua robustez, criando clusters de robustez (CRs) com base na comparação direta entre os coeficientes. Os resultados da classificação mostraram que a seleção de indivíduos das classes de maior robustez devem gerar progênies com menor sensibilidade ambiental, visto que os coeficientes são componentes genéticos aditivos. Conclui-se que a seleção por clusters de robustez é uma forma de amenizar os impactos produzidos nos sistemas de produção por alterações nos ambientes de criação.

Palavras-chave: norma de reação, sensibilidade ambiental, mitigação, mudanças climáticas.

\section{ROBUSTNESS CLUSTERS AS SELECTION CRITERIUM IN GENETIC IMPROVEMENT FOR MITIGATION OF THE IMPACTS OF CLIMATE CHANGE}

\begin{abstract}
Climate changes are expected for the next decades and, consequently, their impacts on cattle breeding, with artificial selection a possible method to mitigate them. This work aimed to develop a selection system based on the genetic parameters generated by adaptive reaction norm models in Nellore cattle. Genetic and growth data were provided by the Brazilian Association of Cattle Breeders. An environmental gradient was created using contemporary group averages standardized to a mean of zero and standard deviation of one. For the prediction of coefficients of the adaptive reaction norms, the random regression model was
\end{abstract}

* raphaifsp@gmail.com 
used, considering cubic polynomials for weights at 450 days with analysis of separated sexes. The genetic values of the different individuals were calculated as a function of the environmental gradient, using the BLUPF90 software. The individuals were classified considering coefficients that generated norms with high genetic values and with lower variation along the environmental gradient. The increase in genetic value and its robustness were then compensated by creating robustness clusters (CRs) based on the direct comparison between the coefficients. The results of the classification showed that the selection of individuals of the classes of greater robustness should generate progenies with less environmental sensitivity, since the coefficients are additive genetic components. It is concluded that the cluster selection of robustness showed to be an effective method of mitigating the impacts produced in the production systems by changes in the breeding environments.

Keywords: reaction norm, environmental sensitivity, mitigation, climatic changes.

\section{INTRODUÇÃO}

Perspectivas de alterações climáticas importantes associadas à crescente concentração de gases do efeito estufa na atmosfera foram apresentadas pelo Painel Intergovernamental de Mudanças Climáticas (IPCC, 2014). De acordo com Denton et al. (2014), as sociedades mundiais precisarão lidar de forma mitigativa e adaptativa para conseguir evitar os impactos nocivos das mudanças no clima, especialmente nas práticas agrícolas e florestais.

Segundo Rojas-Downing et al. (2017), a produção pecuária e, consequentemente, a segurança alimentar serão afetadas pelas alterações no clima. A produção animal nos cenários previstos de mudança ambiental sofrerá impactos conforme ocorram mudanças em fatores produtivos como a disponibilidade de água, a prevalência de doenças e a composição da alimentação animal. Do ponto de vista adaptativo, os mesmos autores concluíram que a utilização de novas linhagens e variedades de animais e culturas agrícolas, bem como o melhoramento genético com objetivos de seleção definidos como tolerância à variabilidade ambiental e a surtos de doenças e pestes são necessários para manter a segurança alimentar. Lallias et al. (2017) colocam a sensibilidade ambiental como característica que reflete a adaptabilidade de populações em diferentes ambientes de criação, podendo ser considerada com critério de seleção para tolerância às mudanças climáticas.

A questão da adaptação genética das populações a novos ambientes se insere no contexto da teoria evolutiva, onde, até recentemente, acreditava-se que o genoma não pudesse sofrer interferências significativas do ambiente, sendo a base da transmissão de características passível de isolamento num modelo linear. Trabalhos relacionados à sensibilidade ambiental foram se tornando relevantes a partir da década de 90, mostrando que o genoma deve ser entendido como dinâmico, sofrendo interação com o ambiente (LEWONTIN, 1998).

Diferentes modelos foram propostos para incorporar a interação entre $o$ genótipo e o ambiente nas pesquisas. Uma possibilidade adotada foi considerar $o$ valor de uma característica fenotípica mensurável em função de um descritor ou gradiente ambiental (GA), sendo tal representação denominada de norma de reação. Tal abordagem foi incorporada aos estudos de evolução e, especialmente, à genética quantitativa, onde o fenótipo foi transformado na predição de valores genéticos ao longo do gradiente ambiental, tornando-se, portanto, normas de reação adaptativas (NRAs). De forma aplicada ao melhoramento genético, o descritor ambiental passou a refletir as variações causadas pelos diferentes sistemas de produção agropecuários, gerando 
resultados imediatos na avaliação da interação entre genótipo e ambiente, até então considerada pouco relevante, especialmente no melhoramento genético animal (DE JONG \& BIJMA, 2002).

Com os modelos de NRAs foi possível observar que, em condições de heterogeneidade ambiental e econômica, a interação genótipo-ambiente (IGA), definida como a expressão diferenciada dos genótipos em diferentes ambientes, permite a seleção de uma propriedade complexa, denominada de plasticidade fenotípica ou sensibilidade ambiental, se em estudos de biologia evolutiva ou de melhoramento genético, respectivamente (RAUW \& GOMEZ-RAYA, 2015). Tal plasticidade fenotípica ocorre com o surgimento de novos fenótipos sem que haja necessariamente um novo alelo: a variação genética pré-existente interage após exposição ao novo parâmetro ambiental, ou seja, a variação fenotípica emerge também da interação entre o componente ambiental e o componente genético (LOFEU \& KOHLSDORF, 2016).

Do ponto de vista da genética quantitativa, a predição de coeficientes para os polinômios que descrevem as curvas de valores genéticos de cada indivíduo tornou possível a seleção de indivíduos que demonstrem robustez na expressão genética, mantendo a produção de sua progênie em diferentes ambientes e sistemas de produção (VANDENPLAS et al., 2013; SORENSEN et al., 2015). Mas a aplicação fora do âmbito teóricoexperimental dos conceitos gerados pelas NRAs ainda não se verificou, visto o direcionamento tecnológico tradicional colocar como objetivo de seleção a maximização da produção sem especificar $\mathrm{o}$ ambiente de criação. Na bovinocultura de

\section{MATERIAL E MÉTODOS}

A pesquisa foi desenvolvida no Instituto Federal de Educação, Ciências e Tecnologia de São Paulo no campus Avaré a partir de dados fornecidos pelo Programa de Melhoramento Genético de Zebuínos, corte, vários trabalhos já foram apresentados, discutindo a existência de interação genótipo-ambiente (IGA) nas populações em seleção das diferentes raças. O Brasil, sendo um país com grande extensão territorial, possui uma gama de sistemas de produção muito diversificada, tornando-se um local de interesse para estudos de IGA por meio dos modelos de NRAs, conforme descrito por Ferreira et al. (2014), Lemos et al. (2015); Hay \& Roberts (2018), Chiaia et al. (2015), Oliveira et al. (2018) e De Araújo Neto et al. (2018).

$\mathrm{Na}$ população de bovinos da raça Nelore, os artigos de Pegolo et al. (2009) e Pegolo et al. (2011) consideraram a possibilidade da seleção nas características de crescimento utilizando como critério de seleção os coeficientes preditos das NRAs, que tendem a ser transmitidos para as proles, permitindo a seleção de indivíduos com robustez ambiental, ou seja, com baixa sensibilidade às mudanças ambientais. O maior desafio associado a tal seleção consiste na padronização dos coeficientes das NRAs num índice que permita a diferenciação dos animais robustos e de baixa produção dos animais robustos e com alta produção, equilibrando o progresso de seleção para ganho de peso com a estabilidade genética.

Este trabalho teve como objetivo desenvolver um critério de seleção baseado nos parâmetros genéticos gerados pela abordagem de norma de reação adaptativa em bovinos da raça Nelore, utilizando modelos de regressão aleatória para a característica de peso aos 450 dias com análise de sexos separados, classificando os animais a serem utilizados com base nos coeficientes das NRAs como critérios de seleção.

numa parceria entre a $\mathrm{ABCZ}$ (Associação Brasileira de Criadores de Zebu) e o IFSP - Campus Avaré. Foram utilizados pesos ajustados de animais aos 450 dias de machos e fêmeas analisados 
separadamente (P40M e P450F, respectivamente) e distribuídos em grupos de contemporâneos (GC), observando-se os efeitos fixos de sexo, estação de nascimento, rebanho e manejo, sendo excluídos os GCs com menos de 40 indivíduos. Os dados analisados estão descritos na Tabela 1.

Tabela 1. Estatística descritiva dos dados analisados para a característica de peso aos 450 dias (P450) considerando dados de Machos e Fêmeas separadamente.

\begin{tabular}{lccccc}
\hline Sexo & N & Média $(\mathbf{k g})$ & DP $(\mathbf{k g})$ & Min $(\mathbf{k g})$ & Max $(\mathbf{k g})$ \\
\hline Machos & 47906 & 279,11 & 50,28 & 109 & 506 \\
Fêmeas & 47058 & 246,17 & 42,14 & 115 & 429 \\
\hline
\end{tabular}

\section{a) Definição do gradiente ambiental}

O descritor ambiental foi calculado utilizando as médias dos GC (PEGOLO et al., 2009) padronizados para uma média zero e um desvio-padrão de um, sendo estes valores multiplicados por $10 \mathrm{e}$ somente a parte inteira considerada, ocasionando um agrupamento dos animais em grupos ambientais (GAs) com valores inteiros. Os valores de GA inferiores a -15 foram considerados no $\mathrm{GA}=-15$ e os valores de GA superiores a 15 foram definidos como $\mathrm{GA}=+15$. Estes valores de GA foram padronizados no intervalo de -1 a 1, para a realização do cálculo dos valores genéticos.

\section{b) Modelo de estimação dos parâmetros}

Utilizou-se o modelo geral, conforme Equação 1 proposta por Pegolo et al. (2011):

$$
y_{i j}=F_{i j}+\sum_{m=\mathbf{0}}^{k_{a \alpha}-\mathbf{1}} \alpha_{i m} \phi_{m}\left(G A_{i j}\right)+\varepsilon_{i j}
$$

em que

$y_{i j}=\mathrm{P} 450$ da j-ésima progênie do i-ésimo animal;

$G A_{i j}=$ grupo ambiental da j-ésima progênie do i-ésimo animal (de -15 a $+15)$;

$\phi_{m}\left(G A_{i j}\right)=$ m-ésimo polinômio de Legendre em função do grupo ambiental $G A_{i j}$;

$F_{i j}=$ efeito fixo de GC;

$\alpha_{i m}=$ coeficiente de regressão aleatória para o efeito genético direto;

$k_{a}=$ ordem do ajuste (definida em nas análise como quatro, considerando

$$
\mathbf{y}=\mathbf{X b}+\mathbf{Z s}+\mathbf{e}
$$

em que

$$
\mathrm{E}\left[\begin{array}{l}
\mathbf{y} \\
\mathbf{s} \\
\varepsilon
\end{array}\right]=\left[\begin{array}{c}
\mathrm{X} \beta \\
0 \\
0
\end{array}\right] \quad \text { e } \quad \mathrm{V}\left[\begin{array}{l}
\mathbf{S} \\
\varepsilon
\end{array}\right]=\left[\begin{array}{cc}
\mathrm{K}_{\mathbf{s}} \otimes \mathbf{A} & \mathbf{0} \\
\mathbf{0} & \mathbf{R}
\end{array}\right]
$$

polinômios de terceiro grau (regressão cúbica), e

$\varepsilon_{i j}=$ efeito do erro associado, com $\mathrm{p}$ classes pré-definidas de variância homogênea.

A partir de análise prévia, verificouse ser possível uma redução das 5 classes residuais para 2 (de -15 a -3 e de -2 a +15 ).

No trabalho, foram definidos polinômios de ordem $\mathrm{m}=4$, ou seja, cúbicos. Assim, foram preditos 4 coeficientes para cada norma de reação adaptativas: de nível $\left(\mathrm{A}_{0}\right)$, de inclinação $\left(A_{1}\right)$, de curvatura $\left(A_{2}\right)$ e de inflexão $\left(A_{3}\right)$.

Na notação matricial:

$\mathbf{y}=$ vetor de observações (P450 das progênies machos e fêmeas, analisadas separadamente);

$\boldsymbol{\beta}=$ vetor de efeitos fixos atribuídos aos grupos contemporâneos;

$s=$ vetor dos coeficientes aleatórios do efeito do animal; 
$\mathbf{X}$ e $\mathbf{Z}=$ as matrizes de incidência correspondentes;

$\boldsymbol{\varepsilon}=$ vetor de resíduos;

$\mathbf{K}_{\mathrm{s}}=$ matriz de coeficientes da função de covariância para o efeito de animal;

$\mathbf{A}=$ matriz de parentesco;

$\mathbf{R}=$ matriz diagonal das variâncias residuais estimadas em duas classes.

Os níveis de $\hat{\sigma}_{(e \mid p)}^{2}$ com $\mathrm{p}=1,2$ foram agrupados nos GAs de -15 a $-3,-2$ a +15 , respectivamente. Estes grupos foram acomodados no modelo de análise pelas matrizes identidade de ordem apropriada para cada nível.

Foram estimados os parâmetros genéticos da população, definidos pela variância genética, variância ambiental e variância fenotípica em cada ponto do gradiente ambiental, gerando herdabilidades também distribuídas ao longo do gradiente. As estimativas de variância residual $\left(\hat{\sigma}_{E}^{z}\right)$ foram obtidas pela diferença entre as estimativas de variância fenotípica $\left(\hat{\sigma}_{P}^{2}=\hat{\sigma}_{S}^{2}+\hat{\sigma}_{(e \mid p)}^{2}\right)$ e a variância aditiva. A herdabilidade foi calculada pela razão entre variância genética e variância fenotípica. Com uma função de (co)variância foram obtidos, para cada animal, os coeficientes dos polinômios de Legendre possibilitando que os valores genéticos fossem encontrados para determinados pontos do GA.

\section{c) Inferência bayesiana}

Estimou-se a matriz de coeficientes da função de (co)variância utilizando-se a metodologia descrita por Pegolo et al. (2011), numa abordagem de normas de reação adaptativas, por meio de regressão aleatória utilizando polinômios de Legendre de quarta ordem (cúbico) numa análise bivariada para sexo (dados de machos e fêmeas analisados separadamente) por inferência bayesiana, que gerou por amostragem de Gibbs 10 000 amostras a cada 100 iterações, em um total de 1050000 iterações e 50000 de "burn-in". Utilizou-se o software GIBBS3F90 ®, pertencente ao pacote BLUPF90 de Misztal (2009), sendo que a matriz de genealogia para a predição dos valores genéticos contou com 412.957 indivíduos. Realizou-se a transformação dos coeficientes dos polinômios de Legendre para coeficientes de polinômios ordinários, gerando os coeficientes da seguinte equação:

$$
\operatorname{VG}(\mid x)=A_{0} x+A_{1} x+A_{2} x^{2}+A_{3} x^{3}
$$

em que

$\mathrm{VG}=$ valor genético do animal;

$\mathrm{A}_{0}=$ coeficiente de intercepto (nível);

$\mathrm{A}_{1}=$ coeficiente de inclinação (linear);

$\mathrm{A}_{2}=$ coeficiente de curvatura (quadrático);

$\mathrm{A}_{3}=$ coeficiente de inflexão (cúbico);

$\mathrm{x}=$ valor do descritor ambiental de variação de $-15 \mathrm{a}+15$ padronizado para $-1 \mathrm{a}+1$.

\section{d) Formação dos clusters de robustez}

Num programa de melhoramento genético utilizando modelos de NRAs, a seleção para o P450 considera os VGs de maior valor em determinado ambiente para identificar os animais com maior potencial de incrementar o progresso de seleção (ganho genético) naquele ambiente. Esta característica está diretamente relacionada ao coeficiente $A_{0}$ da Equação 1. Na seleção para robustez, indivíduos com coeficientes das NRAs que levem a menores variações nos VGs ao longo do gradiente ambiental devem ser selecionados. Neste caso, primeiramente o coeficiente $A_{1}$, e de forma menos intensa, os coeficientes $\mathrm{A}_{2}$ e $\mathrm{A}_{3}$ da equação estão relacionados. Mas, neste último caso, não necessariamente tais indivíduos com baixa sensibilidade ambiental terão VGs mais elevados. Correse o risco de se selecionar indivíduos robustos e que causem uma diminuição do P450. Faz-se necessário utilizar um índice composto, onde ambos os critérios sejam balanceados de forma que se possa identificar animais com valores genéticos elevados e ao mesmo tempo robustos ao longo do gradiente ambiental. O formato escolhido neste trabalho foi se utilizar de 
"clusters" ou grupos de associação entre essas duas características.

Para se agrupar indivíduos nos diferentes clusters de robustez ambiental (CRs) para a característica de P450, considerou-se a necessidade dos seguintes requisitos de classificação: a) melhores animais com maiores valores de $\mathrm{A}_{0}$ (nível); b) melhores animais com $\mathrm{A}_{1}$ (inclinação) tendendo a 0 ; c) melhores animais com valores de $\mathrm{A}_{2}$ (curvatura) positivos ou tendendo a $0 ;$ d) melhores animais com valores de $\mathrm{A}_{3}$ (inflexão) tendendo a 0. Para atender a tais regras, foram gerados os valores padronizados dos coeficientes $\mathrm{Ap}_{0}$, $A p_{1}, A_{2}$ e $A p_{3}$, a partir dos valores médios e os desvios-padrão da distribuição dos valores estimados para cada coeficiente. Foram criados os CLs denominados A, B, $\mathrm{C}, \mathrm{D}$ e não classificado (NC), com níveis cada vez mais relaxados para tais regras, criando-se a distribuição relacionando os valores padronizados de $\mathrm{Ap}_{0}$ e $\mathrm{Ap}_{1}$ em função quadrática específica. A mesma regra foi aplicada aos valores padronizados de $\mathrm{Ap}_{0}$ e $\mathrm{Ap}_{3}$. Exigiu-se um valor mínimo de $\mathrm{Ap}_{2}$, visto haver uma alta correlação deste coeficiente com $\mathrm{Ap}_{0}$. Os limites para cada cluster podem ser observados na Tabela 2 e Figura 1.

Para a formação dos gráficos em três dimensões demonstrando as classificações de robustez em relação ao gradiente ambiental, utilizou-se o software SAS, versão 9.4 para Windows. Copyright (C) 2016 SAS Institute Inc. SAS e todos os outros nomes de produtos e serviços do SAS Institute Inc. são marcas registradas de SAS Institute Inc., Cary, NC, USA.

Tabela 2. Limites para os coeficientes padronizados dos polinômios preditos das NRAs para formação dos clusters de robustez.

\begin{tabular}{ll}
\hline Cluster & Limites \\
\hline A & $\mathrm{Ap}_{0}>4\left(\mathrm{Ap}_{1}\right)^{2}+1,5 ; \mathrm{Ap}_{0}>4\left(\mathrm{Ap}_{3}\right)^{2}+1,5 ; \mathrm{Ap}_{3}>-3$ \\
$\mathrm{~B}$ & $\mathrm{Ap}_{0}>4\left(\mathrm{Ap}_{1}\right)^{2}+1,0 ; \mathrm{Ap}_{0}>4\left(\mathrm{Ap}_{3}\right)^{2}+1,0 ; \mathrm{Ap}_{3}>-4$ \\
$\mathrm{C}$ & $\mathrm{Ap}_{0}>4\left(\mathrm{Ap}_{1}\right)^{2}+0,5 ; \mathrm{Ap}_{0}>4\left(\mathrm{Ap}_{3}\right)^{2}+0.5 ; \mathrm{Ap}_{3}>-5$ \\
$\mathrm{D}$ & $\mathrm{Ap}_{0}>4\left(\mathrm{Ap}_{1}\right)^{2} ; \mathrm{Ap}_{0}>4\left(\mathrm{Ap}_{3}\right)^{2} ; \mathrm{Ap}_{3}>-6$ \\
$\mathrm{NC}$ & $\mathrm{Ap}_{0}<=4\left(\mathrm{Ap}_{1}\right)^{2} ; \mathrm{Ap}_{0}<=4\left(\mathrm{Ap}_{3}\right)^{2} ; \mathrm{Ap} \mathrm{p}_{3}<=-6$ \\
\hline
\end{tabular}

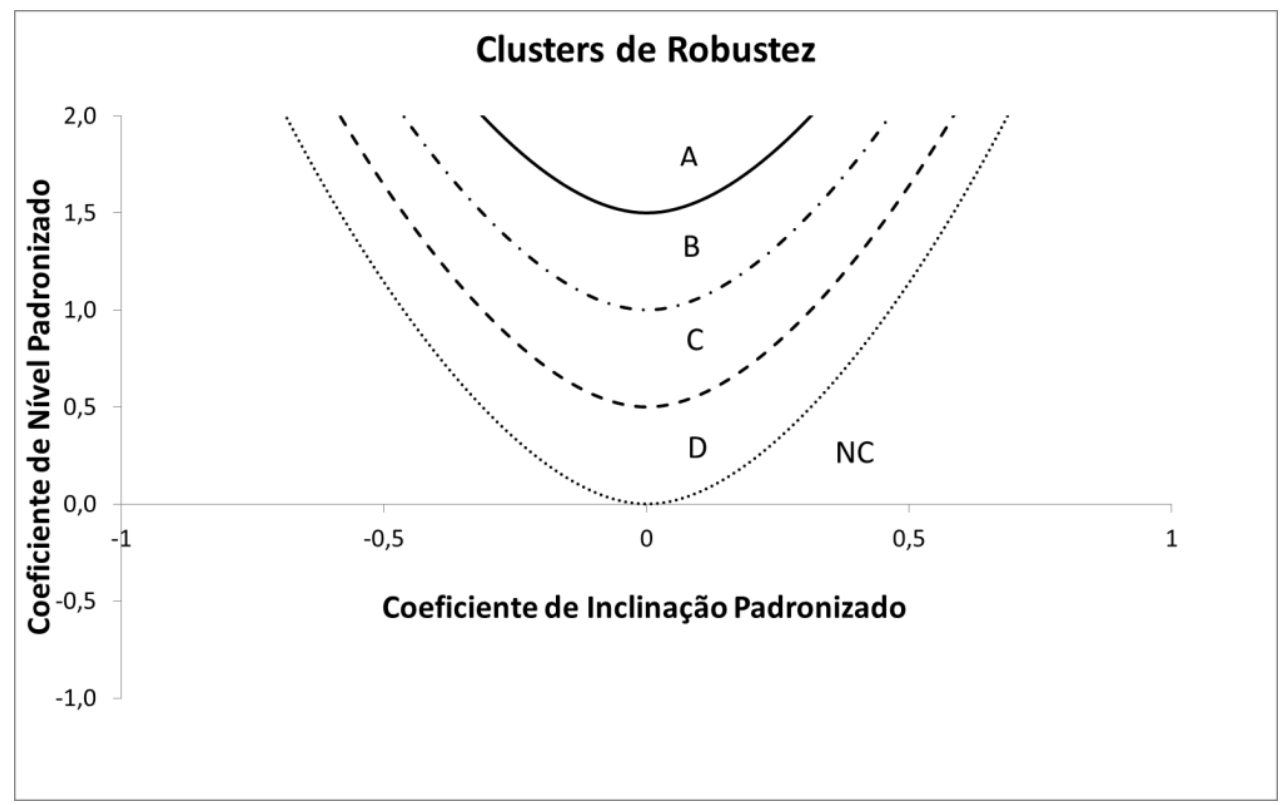

Figura 1. Visualização bidimensional da classificação dos clusters (CL) conforme os Coeficientes de Nível Padronizados e de Inclinação Padronizados, definindo as áreas de $\mathrm{CL}=\mathrm{A}, \mathrm{CL}=\mathrm{B}, \mathrm{CL}=\mathrm{C}, \mathrm{CL}=\mathrm{D}$ e $\mathrm{CL}=\mathrm{NC}$ (não classificado).

RESULTADOS E DISCUSSÃO 
A partir de resultados de análises considerando avaliação genética de rebanhos de gado de corte para a característica peso aos 450 dias, observouse que as herdabilidades (Figura 2) foram menores para os dados oriundos de machos com relação às de fêmeas, especialmente nos extremos ambientais. Também se podem observar os componentes de variância, com valores estimados de variância fenotípica e residuais maiores na análise de dados de machos, havendo também um valor estimado mais elevado da variância genética. Resultados semelhantes foram obtidos por Pegolo et al. (2011), considerando uma população de bovinos Nelore, porém com uma fonte de dados diferenciada.
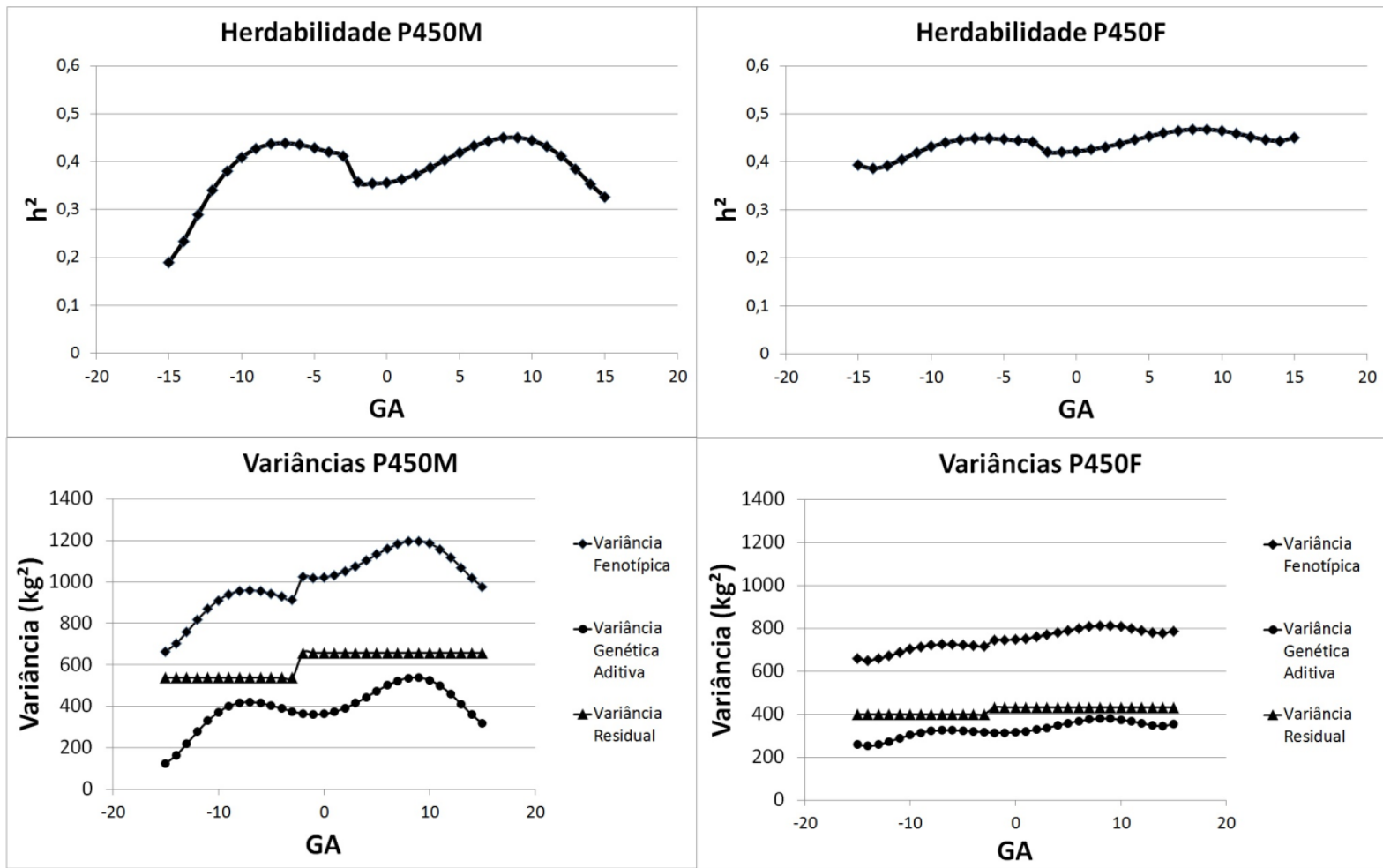

Figura 2. Gráficos dos valores estimados de herdabilidades $\left(h^{2}\right)$ e dos valores estimados das variâncias fenotípicas, genéticas aditivas e residuais, em análises de dados de machos (P450) e de fêmeas (P450F) ao longo do gradiente ambiental (GA).

Os valores estimados de herdabilidades para fêmeas apresentaram curvas mais suaves, com valores maiores e variâncias menores com relação aos machos. Estes apresentaram curvas mais acentuadas com inflexões maiores, conforme se observa na Figura 2, além de valores de herdabilidade mais baixas e variâncias mais altas. Ou seja, dados de machos parecem carregar menos informação relacionada à adaptabilidade que os dados oriundos de fêmeas quando os animais passam a interagir com 0 ambiente em que vivem. O que corrobora com o conceito de conflito entre os sexos (MANK et al., 2014) e os resultados obtidos por Pegolo et al. (2011), comparando os coeficientes de nível e de inclinação estimados. Sugere-se que, como o sexo dos bovinos é definido pelos cromossomos sexuais $\mathrm{XY}$ para machos e XX para fêmeas, a expressão genética para a característica de peso seria resultado de genes posicionados no cromossomo $\mathrm{X}$, transmitido pelo touro para a progênie fêmea. Dessa forma, retomando a teoria evolutiva do sexo, existem hipóteses explicativas para as maiores herdabilidades com dados provenientes em fêmeas, já que estas conservam e transmitem as características; e para as maiores variâncias fenotípicas e residuais para machos, que 
testam os ambientes e permitem a variação genética, possibilitando, para a prole, uma maior adaptação ao ambiente. Os resultados obtidos indicam que padrões reprodutivos considerando regiões nãorecombinantes dos cromossomos sexuais merecem ser foco de novos estudos (SAYRES, 2018), especialmente em sua interação com o ambiente.

Nas análises, foram obtidas correlações genéticas estimadas entre ambientes extremos de 0,33 em fêmeas e 0,40 em machos. Tais valores confirmam a significância da IGA, mostrando a importância do uso de modelos de norma de reação na avaliação de rebanhos bovinos da raça Nelore, em concordância com outros resultados da literatura (PEGOLO et al., 2011; DE ARAÚJO NETO et al., 2018).
Para a visualização dos indivíduos pertencentes a cada um dos CRs, prevendo a seleção futura de animais com menor sensibilidade ambiental, foram criados os gráficos das Figuras 3 e 4, com a representação gráfica dos coeficientes preditos pela função de (co)variância padronizados de cada animal, sendo apresentados somente os indivíduos com um valor mínimo de credibilidade. A Figura 3 exemplifica a distribuição dos valores de análise de machos e a Figura 4, de fêmeas, onde cada ponto corresponde a um animal com os coeficientes de nível padronizados no eixo $\mathrm{y}$, os coeficientes de inclinação padronizados no eixo $\mathrm{x}$ e os coeficientes de inflexão padronizados no eixo z. No caso, utilizou-se o desviopadrão (DP0) da distribuição a posteriori do coeficiente $\mathrm{Ap}_{0}$, apresentando-se os animais com valor menor que 10 .

\section{Análise de İndices de Robustez (M) em função dos Coeficientes}

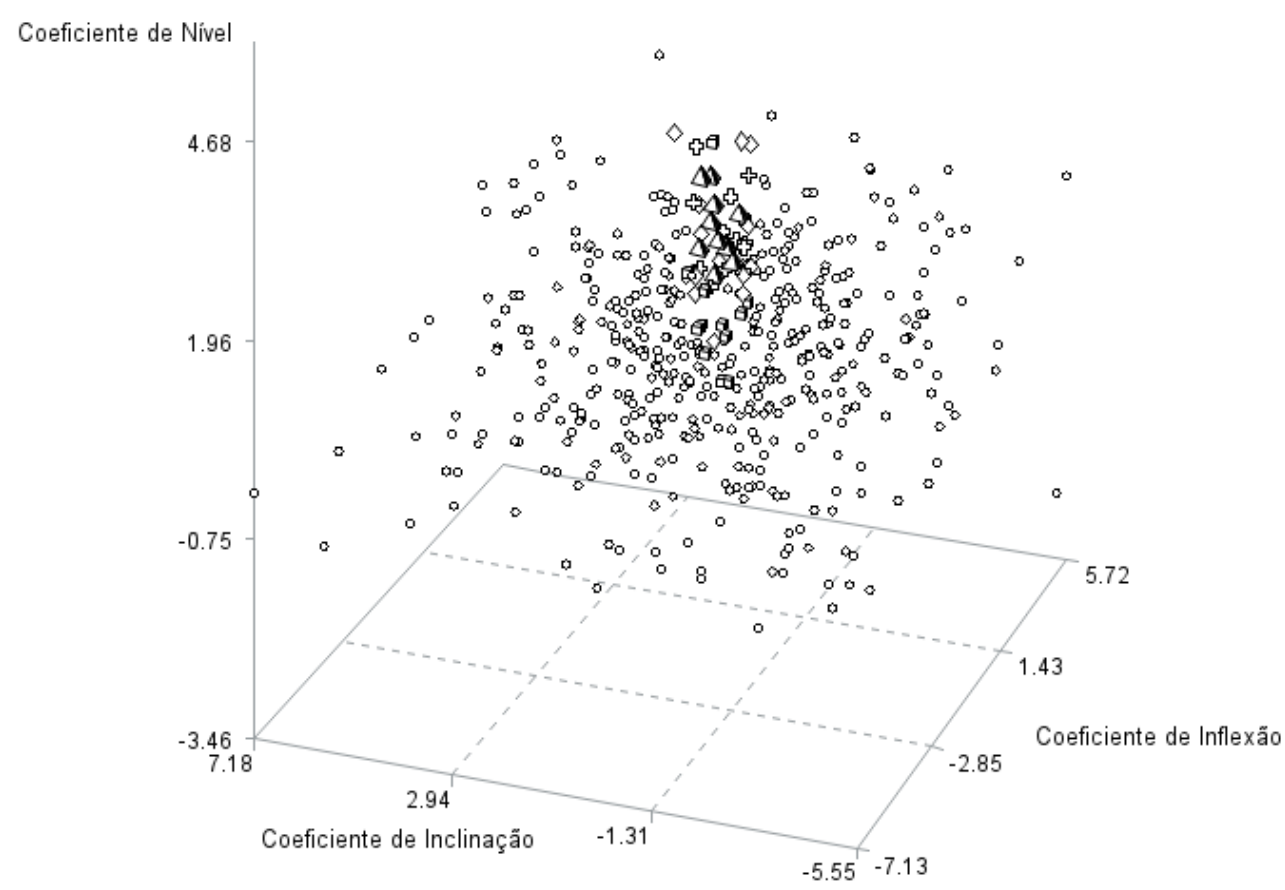

Figura 3. Representação tridimensional dos valores dos coeficientes preditos para dados de Machos. No Cluster de Robustez: $(\mathrm{CR})=\mathrm{A}$ estão representados por pirâmides; $\mathrm{CR}=\mathrm{B}$ por cruzes; $\mathrm{CR}=\mathrm{C}$ por diamantes; $\mathrm{CR}=\mathrm{D}$ por cubos e não Classificados $(\mathrm{CR}=\mathrm{NC})$ por balões. 


\section{Análise de İ́ndices de Robustez (F) em função dos Coeficientes}

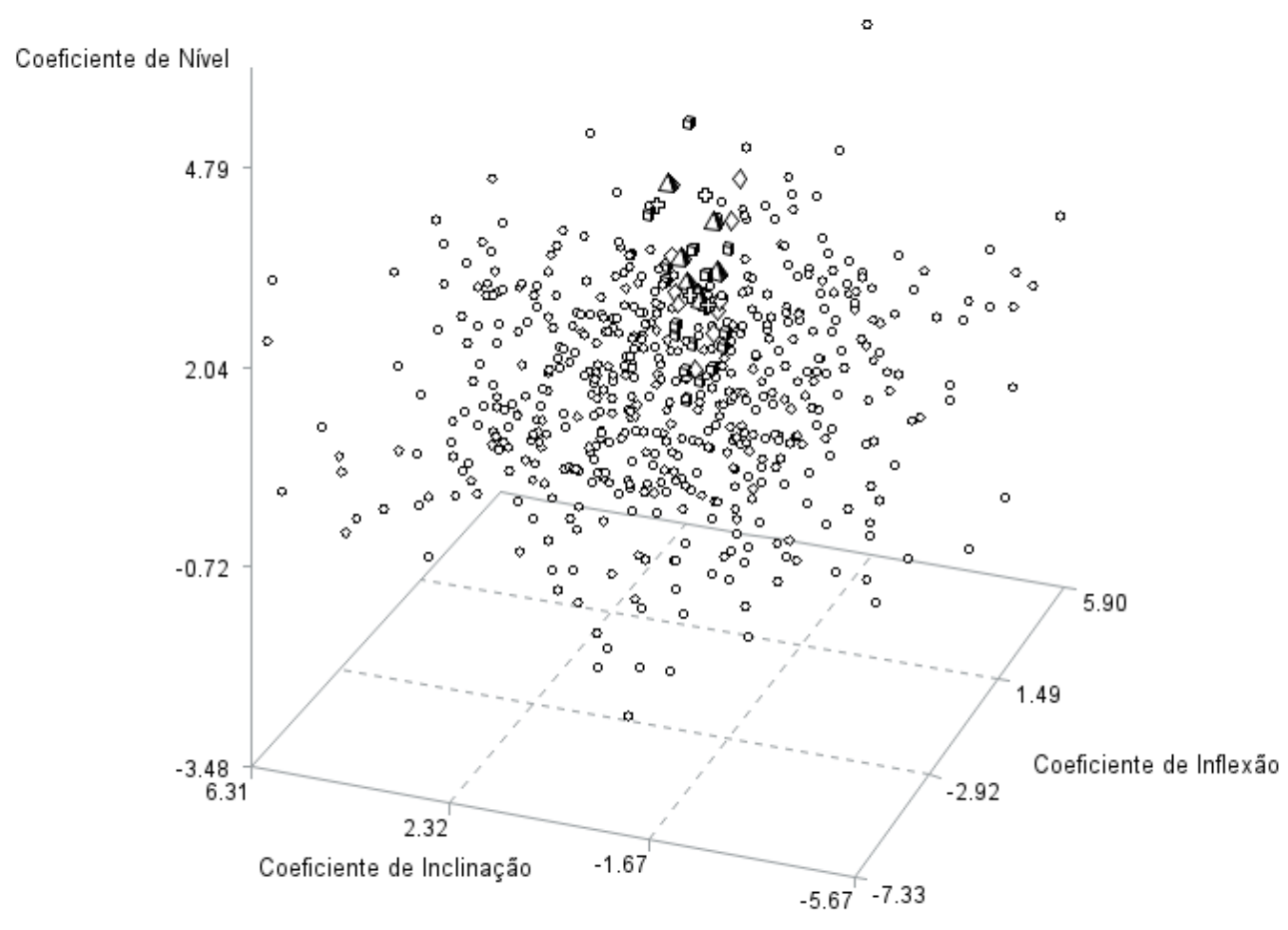

Figura 4. Representação tridimensional dos valores dos coeficientes preditos para dados de fêmeas. No Cluster de Robustez: $(C R)=A$ estão representados por pirâmides; $C R=B$ por cruzes; $C R=C$ por diamantes; $C R=D$ por cubos e não Classificados $(C R=N C)$ por balões.

Observa-se, pela Tabela 3, que foi realizada a classificação dos animais utilizando os coeficientes que, concomitantemente, gerassem normas com valores genéticos elevados e com menor variação ao longo do gradiente ambiental. O método permitiu uma compensação entre a elevação do valor genético e a sua robustez, criando clusters de robustez (CLs) com base na comparação direta entre os coeficientes, ao invés de limites constantes. $\mathrm{O} C \mathrm{CL}=\mathrm{A}$ teve amplitude de coeficiente de inclinação e deflexão menores que $\mathrm{CL}=\mathrm{B}$, sendo que $\mathrm{CL}=\mathrm{B}$ teve tais amplitudes menores que $\mathrm{CL}=\mathrm{C}$, e assim por diante para $\mathrm{CL}=\mathrm{D}$ e $\mathrm{NC}$. Os valores médios dos coeficientes de nível foram sempre decrescentes de $\mathrm{CL}=\mathrm{A}$ para $\mathrm{CL}=\mathrm{NC}$. É possível notar tais valores decrescentes de $\mathrm{A}_{0}$ a cada classificação mais baixa, tanto para machos quanto para fêmeas, demonstrando sua relação com o sistema de classificação de animais. 
Tabela 3. Estatística descritiva dos diferentes Grupos de Robustez (GR=A, B, C e D), de animais com desvios-padrão da distribuição "a posteriori” menores que 10.

\begin{tabular}{|c|c|c|c|c|c|c|c|c|c|c|c|}
\hline \multirow[t]{2}{*}{ GR } & \multicolumn{2}{|c|}{$\mathbf{N}^{\circ}$ OBS } & \multirow[t]{2}{*}{ COEFICIENTES } & \multicolumn{2}{|c|}{ MÉDIA } & \multicolumn{2}{|c|}{ DP } & \multicolumn{2}{|c|}{ MÍN. } & \multicolumn{2}{|c|}{ MÁX. } \\
\hline & M & $\mathbf{F}$ & & $\mathbf{M}$ & $\mathbf{F}$ & $\mathbf{M}$ & $\mathbf{F}$ & $\mathbf{M}$ & $\mathbf{F}$ & $\mathbf{M}$ & $\mathbf{F}$ \\
\hline \multirow{5}{*}{ A } & \multirow{5}{*}{10} & \multirow{5}{*}{7} & A0 & 27,90 & 25,75 & 4,33 & 5,58 & 21,12 & 20,39 & 34,53 & 35,99 \\
\hline & & & A1 & $-0,71$ & 1,78 & 2,47 & 1,89 & $-4,66$ & $-0,78$ & 2,28 & 4,55 \\
\hline & & & A2 & $-9,08$ & $-3,35$ & 2,38 & 4,07 & $-12,53$ & $-7,86$ & $-5,71$ & 1,45 \\
\hline & & & A3 & 2,95 & $-1,45$ & 1,60 & 0,89 & 0,37 & $-2,81$ & 6,24 & 0,08 \\
\hline & & & DP0 & 6,56 & 6,67 & 2,21 & 2,14 & 2,96 & 3,04 & 9,67 & 8.76 \\
\hline \multirow{5}{*}{ B } & \multirow{5}{*}{13} & \multirow{5}{*}{6} & A0 & 26,09 & 24,57 & 5,56 & 6,77 & 19,99 & 19,01 & 36,91 & 33,81 \\
\hline & & & $\mathrm{A} 1$ & $-1,92$ & 1,56 & 3,52 & 2,29 & $-5,53$ & $-0,63$ & 4,99 & 5,96 \\
\hline & & & A2 & $-8,46$ & $-1,30$ & 3,59 & 5,40 & $-15,23$ & $-10,15$ & $-2,04$ & 4,17 \\
\hline & & & A3 & 4,12 & $-2,01$ & 2,50 & 1,43 & 0,36 & $-3,44$ & 7,68 & 0,11 \\
\hline & & & DP0 & 6,85 & 6,56 & 2,63 & 2,75 & 2,27 & 3,34 & 9,26 & 9,90 \\
\hline \multirow{5}{*}{ C } & \multirow{5}{*}{13} & \multirow{5}{*}{11} & A0 & 25,44 & 20,11 & 8,89 & 7,27 & 11,31 & 10,00 & 41,92 & 34,85 \\
\hline & & & A1 & $-0,94$ & 1,25 & 4,31 & 2,66 & $-6,13$ & $-2,29$ & 4,90 & 5,36 \\
\hline & & & A2 & $-7,48$ & 0,15 & 6,01 & 5,54 & $-18,62$ & $-9,61$ & 0,47 & 9,06 \\
\hline & & & A3 & 4,34 & $-0,36$ & 3,63 & 1,33 & $-3,56$ & $-2,87$ & 8,98 & 1,99 \\
\hline & & & DP0 & 6,50 & 8,46 & 2,03 & 1,26 & 3,60 & 5,48 & 9,70 & 9,73 \\
\hline \multirow{5}{*}{ D } & \multirow{5}{*}{15} & \multirow{5}{*}{16} & A0 & 16,78 & 19,58 & 8,60 & 9,78 & 5,91 & 7,25 & 40,17 & 42,43 \\
\hline & & & A1 & $-0,30$ & 2,09 & 3,11 & 2,97 & $-5,25$ & $-2,92$ & 4,24 & 6,51 \\
\hline & & & A2 & $-4,54$ & $-1,72$ & 5,74 & 7,01 & $-22,43$ & $-17,70$ & 1,10 & 10,52 \\
\hline & & & A3 & 3,38 & $-1,79$ & 2,81 & 1,69 & $-1,87$ & $-5,25$ & 8,09 & $-0,01$ \\
\hline & & & DP0 & 7,30 & 7,18 & 1,93 & 2.05 & 3,04 & 3,07 & 9,90 & 9,89 \\
\hline \multirow{5}{*}{ NC } & \multirow{5}{*}{431} & \multirow{5}{*}{526} & A0 & 9,55 & 8,80 & 13,27 & 12,49 & $-29,19$ & $-27,84$ & 50,97 & 48,99 \\
\hline & & & A1 & $-0,43$ & 3,72 & 19,14 & 12,81 & $-53,45$ & $-33,57$ & 65,57 & 40,84 \\
\hline & & & A2 & $-1,16$ & 1,55 & 6,44 & 6,44 & $-19,18$ & $-19,87$ & 19,27 & 21,15 \\
\hline & & & $\mathrm{A} 3$ & 2,77 & $-3,55$ & 16,00 & 10,14 & $-53,70$ & $-36,58$ & 47,58 & 26,40 \\
\hline & & & DP0 & 7,34 & 7,14 & 1,87 & 2,01 & 2,54 & 2,02 & 10,00 & 9,99 \\
\hline
\end{tabular}

\section{CONCLUSÕES}

Como os coeficientes estimados pelos modelos de norma de reação adaptativa são componentes genéticos aditivos, concluiu-se que estes afetam diretamente as características da progênie gerada. Animais selecionados por estar em clusters de robustez superiores devem gerar progênies com menor sensibilidade ambiental, sofrendo menores alterações com mudanças nos ambientes de criação. Concluiu-se que a seleção de animais por clusters de robustez é possível através de coeficientes de normas de reação adaptativa, podendo amenizar os impactos nos sistemas de produção por alterações nos ambientes de criação.

\section{AGRADECIMENTOS}

Os autores agradecem às instituições de financiamento e fomento do projeto: $\mathrm{CNPq}$, ABCZ e Núcleo Nelore Avaré.

\section{REFERÊNCIAS BIBLIOGRÁFICAS}

CHIAIA, H. L. J., DE LEMOS, M. V. A., VENTURINI, G. C., ABOUJAOUDE, C., BERTON, M. P., FEITOSA, F. B., CARVALHEIRO, R., ALBUQUERQUE, L. G., DE OLIVEIRA, H. N., BALDI, F. Genotype $\mathrm{x}$ environment interaction for age at first calving, scrotal circumference, and yearling weight in Nellore cattle using reaction norms in multitrait random regression models, Journal of Animal Science, v, 93, n.4, p.1503-1510, 2015.

DE ARAÚJO NETO, F.R.; PEGOLO, N.T.; ASPILCUETA-BORQUIS, R.R.; PESSOA, M.C.; BONIFÁCIO, A.; LOBO, R.B.; DE OLIVEIRA, H.N. Study of the effect of genotype-environment interaction on age at first calving and production traits 
in Nellore cattle using multi-trait reaction norms and Bayesian inference. Animal Science Journal, 2018. No prelo. Disponível em: https://onlinelibrary.wiley.com/doi/pdf/10. 1111/asj.12994. Acesso em: 30 mai. 2018.

DE JONG, G.; BIJMA, P. Selection and phenotypic plasticity in evolutionary biology and animal breeding. Livestock Production Science, v.78, n. 3, p.195-214, 2002.

DENTON, F.; WILBANKS, T. J.; ABEYSINGHE, A. C.; BURTON, I.; GAO, Q.; LEMOS, M. C.; MASUI, T.; O'BRIEN, K. L.; WARNER, K. Climateresilient pathways: adaptation, mitigation, and sustainable development. Climate Change 2014: Impacts, Adaptation, and Vulnerability, p. 1101-1131, 2014.

FERREIRA, J. L.; LOPES, F. B.; ASSIS, A. S.; LÔBO, R. B. Espacialização climática e análise de dados longitudinais de bovinos Nelore criados nos Estados do Maranhão, Pará e Tocantins por meio de técnicas univariadas e multivariadas. Semina: Ciências, Agrárias, Londrina, v. 35, n. 4, p. 2197-2210, 2014.

HAY, E. H.; ROBERTS A. Genotype $\times$ prenatal and post-weaning nutritional environment interaction in a composite beef cattle breed using reaction norms and a multi-trait model. Journal of Animal Science, v.96, n. 2, p.444-453, 2018.

\section{IPCC - INTERGOVERNMENTAL PANEL ON CLIMATE CHANGE. Climate Change 2014- Impacts, Adaptation and Vulnerability: Regional Aspects. Cambridge University Press, 2014.}

LALLIAS, D.; QUILLET, E.; BÉGOUT, M.-L.; AUPÉRIN, B.; KHAW, H. L.; MILLOT, S.; VALOTAIRE, C.; KERNÉIS, T.; LABBÉ, L.; PRUNET, P; DUPONT-NIVET, M. Genetic variability of environmental sensitivity revealed by phenotypic variation in body weight and (its) correlations to physiological and behavioral traits. PLoS One, v.12, n.12, 2017.

LEMOS, M. V. A.; CHIAIA, H. L. J.; BERTON, M.; FEITOSA, F. L. B.; ABOUJAOUDE, C.; VENTURINI, G. C.; DE OLIVEIRA, H. N.; DE ALBUQUERQUE, L. G.; BALDI, F. Reaction norms for the study of genotypeenvironment interaction for growth and indicator traits of sexual precocity in Nellore cattle. Genetics and Molecular Research, v. 14, n. 2, p. 7151-7162, 2015.

LEWONTIN, R. C. The Triple Helix: Gene, Organism an. Environment. Harvard University Press, Cambridge. 1998. 192p. LOFEU, L.; KOHLSDORF, T. Mais que seleção: o papel do ambiente na origem e evolução da diversidade fenotípica. $1^{\text {a }}$ edição. Ribeirão Preto: Sociedade Brasileira de Genética, v. 10, n.1, p. 1119, 2016.

MANK, J. E.; HOSKEN, D. J.; WEDELL, N. Conflict on the sex chromosomes: cause effect and complexity. Cold Spring Harbor Perspectives in Biology, v.6, n.12, a017715, 2014.

MISZTAL, I. BLUP90 family of programs. 2009. Disponível em: $<$ http://nce.ads.uga.edu/ ignacy/newprogra ms.html>. Acesso em: 19 de jun. 2017.

OLIVEIRA, D.P.; LOURENÇO, D. A. L.; TSURUTA, S.; MISZTAL, I.; SANTOS, D. J. A.; DE ARAÚJO NETO, F. R.; ASPILCUETA-BORQUIS, R. R.; BALDI, F.; CARVALHEIRO, R.; DE CAMARGO, G. M. F.; ALBUQUERQUE, L. G.; TONHATI, H. Reaction norm for yearling weight in beef cattle using single-step genomic evaluation. Journal of Animal Science, v. 96, p.27-34, 2018.

PEGOLO, N. T.; OLIVEIRA, H. N.; ALBUQUERQUE, L. G.; BEZERRA, L. A. F.; LÔBO, R. B. Genotype by environment interaction for 450-day weight of Nelore cattle analyzed by 
reaction norm models. Genetics and Molecular Biology. São Paulo, v. 32, n. 2, p. 281-287, 2009.

PEGOLO, N. T., ALBUQUERQUE, L. G. D., LÔBO, R. B., \& DE OLIVEIRA, H. N. Effects of sex and age on genotypex environment interaction for beef cattle body weight studied using reaction norm models. Journal of animal science, v. 89, n. 11, p. 3410-3425, 2011.

RAUW, W. M.; GOMEZ-RAYA, L. Genotype by environment interaction and breeding for robustness in livestock. Frontiers in genetics, v. 6, p. 310, 2015.

ROJAS-DOWNING M. M.; NEJADHASHEMI, A. P.; HARRIGAN, T.; WOZNICKI, S. A. Climate change and livestock: Impacts, adaptation, and mitigation. Science Direct, v. 16, p. 145163, 2017.

SAYRES, M. A. W. Genetic Diversity on the Sex Chromosomes, Genome Biology and Evolution, v.10,n. 4, p.1064-1078, 2018.

SORENSEN P.; DE LOS CAMPOS, G.; MORGANTE, F.; MACKAY, T.; SORENSEN, D. Genetic Control of Environmental Variation of Two Quantitative Traits of Drosophila melanogaster Revealed by Whole-Genome Sequencing. Genetics.v. 201, n. 2,p.487497, 2015.

VANDENPLAS, J.; BASTIN, C., GENGLER, N., MULDER, H. A.Genetic variance in micro-environmental sensitivity for milk and milk quality in Walloon Holstein cattle. Journal Dairy Science. v.96, p.5977-5990, 2013. 